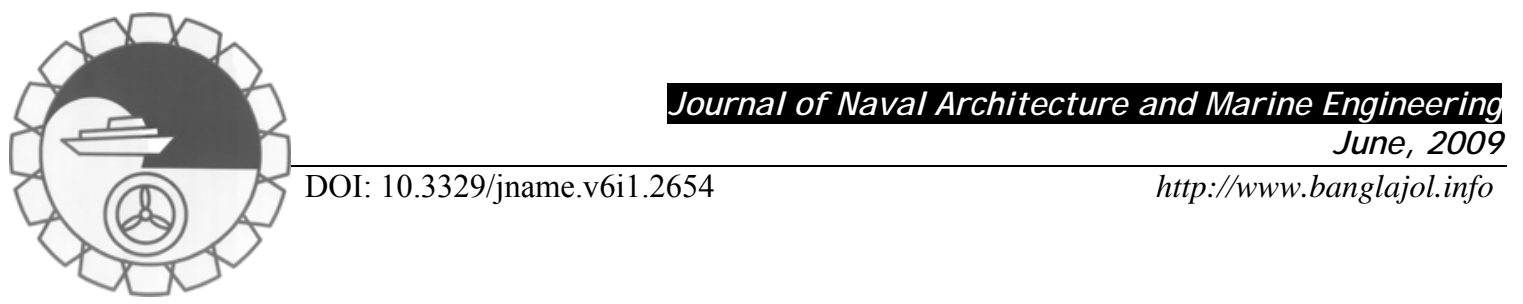

\title{
NUMERICAL STUDY OF MAGNETOHYDRODYNAMC FREE CONVECTIVE HEAT TRANSFER FLOW ALONG A VERTICAL FLAT PLATE WTH TEMPERATURE DEPENDENT THERMAL CONDUCTIMTY
}

\author{
M. M. Rahman ${ }^{1}$ and M. A. Alim ${ }^{2}$ \\ ${ }^{1}$ Department of Computer Science and Engineering, Dhaka International University, Banani, Dhaka -1213, Bangladesh, \\ Email: mrrahmandiu@yahoo.com \\ ${ }^{2}$ Department of Mathematics, Bangladesh University of Engineering and Technology, Dhaka-1000, Bangladesh, Email: \\ maalim@math.buet.ac.bd
}

\begin{abstract}
:
The present numerical work describes the effect of the magnetohydrodynamic (MHD) free convective heat transfer flow along a vertical flat plate with temperature dependent thermal conductivity and heat conduction. The governing equations reduce to local non-similarity boundary layer equations using suitable transformation have been integrated by employing an implicit finite difference method together with the Keller box technique. Comparison with previously published work is performed and excellent agreement is observed. Profiles of the dimensionless velocity and temperature distributions as well as the local skin friction coefficient and surface temperature distribution are shown graphically for various values of the magnetic parameter $M$, thermal conductivity variation parameter $\gamma$ and Prandt number Pr.
\end{abstract}

Keywords: Implicit finite difference method, free convection flow, vertical flat plate, temperature dependent thermal conductivity.

\begin{tabular}{|c|c|c|c|}
\hline \multicolumn{2}{|c|}{ NOMENCLATURE } & \multicolumn{2}{|c|}{ Greek symbols } \\
\hline$b$ & plate thickness & $\bar{u}, \bar{v}$ & velocity components \\
\hline$C_{f x}$ & local skin friction coefficient & $u, v$ & dimensionless velocity components \\
\hline$c_{p}$ & specific heat at constant pressure & $\bar{x}, \bar{y}$ & cartesian coordinates \\
\hline$f$ & dimensionless stream function & $x, y$ & dimensionless cartesian coordinates \\
\hline$g$ & acceleration due to gravity & $\beta$ & co-efficient of thermal expansion \\
\hline$G r$ & Grashof number & $\nabla$ & vector differential operator \\
\hline$h$ & dimensionless temperature & $\eta$ & dimensionless similarity variable \\
\hline$H_{0}$ & strength of the magnetic field & $\theta(x, 0)$ & surface temperature distribution \\
\hline$l$ & length of the plate & $\kappa_{f}$ & thermal conductivity of the fluid \\
\hline$M$ & magnetic parameter & $\kappa_{s}$ & thermal conductivity of the solid \\
\hline$\gamma$ & thermal conductivity variation parameter & $\kappa_{\infty}$ & thermal conductivity of the ambient fluid \\
\hline$P$ & conjugate conduction parameter & $\mu, v$ & dynamic and kinematic viscosities \\
\hline $\operatorname{Pr}$ & Prandtl number & $\rho$ & density of the fluid \\
\hline$T$ & temperature of the interface & $\sigma$ & electrical conductivity \\
\hline$T_{\mathrm{b}}$ & temperature at outside surface of the plate & $\tau_{w}$ & shearing stress \\
\hline$T_{f}$ & temperature of the fluid & $\psi$ & stream function \\
\hline$T_{\infty}$ & fluid asymptotic temperature & & \\
\hline
\end{tabular}




\section{Introduction}

The most common type of body force, which acts on a fluid is due to gravity so that the body force can be defined as in magnitude and direction by the acceleration due to gravity. The electric and magnetic fields themselves must obey a set of physical laws, which are expressed by Maxwell's equations. The solution of such problems requires the simultaneous solution of the equations of fluid mechanics and of electromagnetism. One special case of this type of coupling is the field known as MHD. The interaction of the magnetic field and the moving electric charge carried by the flowing fluid induces a force, which tends to oppose the fluid motion. And near the leading edge the velocity is very small so that the magnetic force, which is proportional to the magnitude of the longitudinal velocity and acts in the opposite direction, is also very small. Consequently, the influence of the magnetic field on the boundary layer is exerted only through induced forces within the boundary layer itself, with no additional effects arising from the free stream pressure gradient. Magnetohydrodynamic flow is an important research area due to its potential applications in engineering and industrial fields. Magnetohydrodynamic power generators and accelerators, cooling of nuclear reactors and crystal growth are included in this area. Accordingly, a considerable amount of research has been accomplished on the effects of electrically- conducting fluids such as liquid metals water mixed with a little acid and others in the presence of transverse magnetic field on the flow and heat transfer characteristics over various geometries. The effect of magnetic field on free convection heat transfer has studied by Sparrow and Cess (1961). Kuiken (1970) studied the problem of MHD free convection in a strong cross field. Hossain et al. (1990, 1997, and 1998) discussed the both forced and free convection boundary layer flow of an electrically conducting fluid in the presence of magnetic field. Moreover, MHD free convection flow of visco-elastic fluid past an infinite porous plate was investigated by Chowdhury and Islam (2000). Elbashbeshy (2000) also discussed the effect of free convection flow with variable viscosity and thermal diffusivity along a vertical plate in the presence of magnetic field.

In all the above studies the effects of temperature dependent thermal conductivity has not been considered. But, thermal boundary layer in liquid metals with variable thermal conductivity studied by Arunachalam and Rajappa (1978). The Combined convection from a vertical flat plate with temperature dependent viscosity and thermal conductivity was investigated by Hossain et al. (2002). Hossain et al. (2004) have considered the problem of the natural convection laminar flow with temperature dependent viscosity and thermal conductivity along a vertical wavy surface. Moreover, the natural convection flow from an isothermal sphere with temperature dependent thermal conductivity has studied by Molla et al. (2005).

Therefore the objective of the present work is to investigate the numerical study on MHD free convection flow along a vertical flat plate with temperature dependent thermal conductivity and heat conduction. In our study, we have considered the conductivity of the fluid to be proportional to a linear function of temperature as considered by Charraudeau (1975) .The governing partial differential equations are reduced to locally nonsimilar partial differential forms by adopting appropriate transformations. The transformed boundary layer equations are solved numerically using very efficient finite difference scheme known as Keller box technique (1978). Numerical results of the velocity, temperature, local skin friction coefficient and the surface temperature distribution for the thermal conductivity variation parameter, the magnetic parameter and Prandtl number are presented graphically.

\section{Mathematical Formulation}

We consider a steady two-dimensional laminar free convection flow of an electrically conducting, viscous and incompressible fluid along a vertical flat plate of length $l$ and thickness $b$. It is assumed that the temperature at the outer surface of the plate is maintained at a constant temperature $T_{b}$, where $T_{b}>T_{\infty}$. Here $T_{\infty}$ the temperature of the fluid outside the boundary layer. The coordinates system and the configuration are shown in Fig. 1

The governing equations for continuity, momentum and energy take the following form

$$
\begin{aligned}
& \frac{\partial \bar{u}}{\partial \bar{x}}+\frac{\partial \bar{v}}{\partial \bar{y}}=0 \\
& \bar{u} \frac{\partial \bar{u}}{\partial \bar{x}}+\bar{v} \frac{\partial \bar{u}}{\partial \bar{y}}=v \frac{\partial^{2} \bar{u}}{\partial \bar{y}^{2}}+g \beta\left(T_{f}-T_{\infty}\right)-\frac{\sigma H_{0}^{2} \bar{u}}{\rho}
\end{aligned}
$$




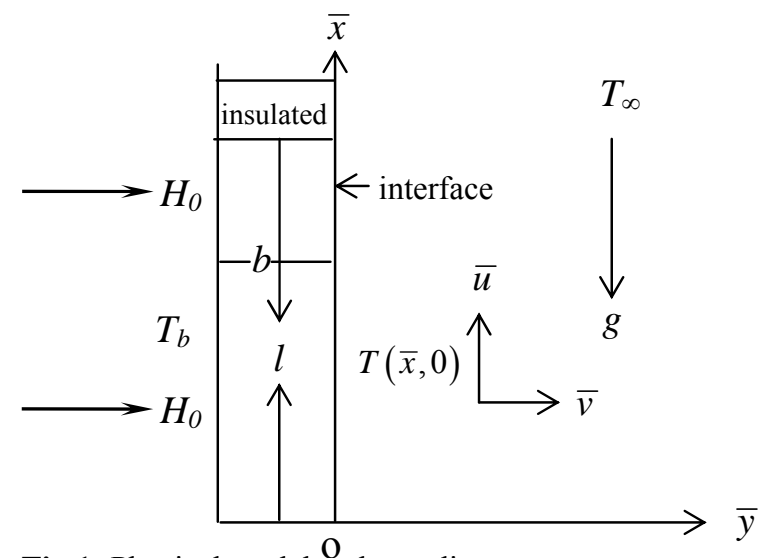

Fig.1: Physical model and coordinate system

$\bar{u} \frac{\partial T_{f}}{\partial \bar{x}}+\bar{v} \frac{\partial T_{f}}{\partial \bar{y}}=\frac{1}{\rho c_{p}} \frac{\partial}{\partial \bar{y}}\left(\kappa_{f} \frac{\partial T_{f}}{\partial \bar{y}}\right)$

Here we will consider the form of the temperature dependent thermal conductivity, which is proposed by Charraudeau [1975]

$\kappa_{f}=\kappa_{\infty}\left[1+\delta\left(T_{f}-T_{\infty}\right)\right]$

where $\kappa_{\infty}$ is the thermal conductivity of the ambient fluid and $\delta$ is a constant.

The appropriate boundary conditions to be satisfied by the above equations are (Luikov 1974, Merkin \& Pop 1996, Pop \& Ingham 2001, Cheng 2006)

$$
\left.\begin{array}{l}
\bar{u}=0, \quad \bar{v}=0 \\
T_{f}=T(\bar{x}, 0), \quad \frac{\partial T_{f}}{\partial \bar{y}}=\frac{\kappa_{s}}{b \kappa_{f}}\left(T_{f}-T_{b}\right) \quad \text { at } \bar{y}=0, \bar{x}>0 \\
\bar{u} \rightarrow 0, T_{f} \rightarrow T_{\infty} \text { as } \quad \bar{y} \rightarrow \infty, \quad \bar{x}>0
\end{array}\right\}
$$

The non-dimensional governing equations and boundary conditions can be obtained from Equations (1) - (5) using the following non-dimensional quantities

$$
\begin{aligned}
& x=\frac{\bar{x}}{l}, y=\frac{\bar{y}}{l} G r^{\frac{1}{4}}, u=\frac{\bar{u} l}{v} G r^{-\frac{1}{2}}, \\
& v=\frac{\bar{v} l}{v} G r^{-\frac{1}{4}}, \theta=\frac{T_{f}-T_{\infty}}{T_{b}-T_{\infty}}, \\
& G r=\frac{g \beta l^{3}\left(T_{b}-T_{\infty}\right)}{v^{2}}
\end{aligned}
$$

where $l$ is the length of the plate, $G r$ is the Grashof number, $\theta$ is the non-dimensional temperature.

Equations (1) to (3) we get the following non -dimensional equations

$$
\frac{\partial u}{\partial x}+\frac{\partial v}{\partial y}=0
$$


$u \frac{\partial u}{\partial x}+v \frac{\partial u}{\partial y}+M u=\frac{\partial^{2} u}{\partial y^{2}}+\theta$

$u \frac{\partial \theta}{\partial x}+v \frac{\partial \theta}{\partial y}=\frac{1}{\operatorname{Pr}}(1+\gamma \theta) \frac{\partial^{2} \theta}{\partial y^{2}}+\frac{\gamma}{\operatorname{Pr}}\left(\frac{\partial \theta}{\partial y}\right)^{2}$

where $\operatorname{Pr}=\left(\mu c_{p}\right) / k_{\infty}$ is the Prandtl number, $\gamma=\delta\left(T_{b}-T_{\infty}\right)$, is the dimensionless thermal conductivity variation parameter and $M=\sigma H_{0}^{2} l^{2} / \mu G r^{1 / 2}$ is the dimensionless magnetic parameter.

The corresponding boundary conditions (5) then take the following form

$u=0, v=0, \theta-1=(1+\gamma \theta) p \frac{\partial \theta}{\partial y} \quad$ at $\left.y=0, x>0 \quad\right\}$

$u \rightarrow 0, \theta \rightarrow 0 \quad$ as $y \rightarrow \infty, x>0$

where $p=\left(k_{\infty} b\right) /\left(k_{s} l\right) G r^{1 / 4}$ is the conjugate conduction parameter. This coupling parameter determines the significance of the conduction resistance within the wall. In the present investigation we have considered $p=1$.

To solve the Equations (8) and (9) subject to the boundary conditions (10) the flowing transformations are then introduced

$$
\begin{aligned}
& \psi=x^{\frac{4}{5}}(1+x)^{\frac{1}{-20}} f(x, \eta), \\
& \eta=y x^{-\frac{1}{5}}(1+x)^{-\frac{1}{20}}, \\
& \theta=x^{\frac{1}{5}}(1+x)^{-\frac{1}{5}} h(x, \eta)
\end{aligned}
$$

here $\eta$ is the similarity variable and $\psi$ is the non-dimensional stream function which satisfies the continuity equation and is related to the velocity components in the usual way as $u=\partial \psi / \partial y$ and $v=-\partial \psi / \partial x$. Moreover $h(x, \eta)$ represents the dimensionless temperature. The momentum and energy equations (Equation (8) and (9), respectively) are transformed for the new coordinate system. Thus we get

$$
\begin{aligned}
& f^{\prime \prime \prime}+\frac{16+15 x}{20(1+x)} f f^{\prime \prime}-\frac{6+5 x}{10(1+x)} f^{\prime 2}-M x^{\frac{2}{5}}(1+x)^{\frac{1}{10}} f^{\prime}+h=x\left(f^{\prime} \frac{\partial f^{\prime}}{\partial x}-f^{\prime \prime} \frac{\partial f}{\partial x}\right) \\
& \frac{1}{\operatorname{Pr}} h^{\prime \prime}+\frac{\gamma}{\operatorname{Pr}}\left(\frac{x}{1+x}\right)^{\frac{1}{5}} h h^{\prime \prime}+\frac{\gamma}{\operatorname{Pr}}\left(\frac{x}{1+x}\right)^{\frac{1}{5}} h^{\prime 2}+\frac{16+15 x}{20(1+x)} f h^{\prime}-\frac{1}{5(1+x)} f^{\prime} h \\
& =x\left(f^{\prime} \frac{\partial h}{\partial x}-h^{\prime} \frac{\partial f}{\partial x}\right)
\end{aligned}
$$

where prime denotes partial differentiation with respect to $\eta$. The boundary conditions as mentioned in Equation (10) then take the following form

$$
\begin{aligned}
& f(x, 0)=f^{\prime}(x, 0)=0, \\
& h^{\prime}(x, 0)=\frac{x^{\frac{1}{5}}(1+x)^{-\frac{1}{5}} h(x, 0)-1}{(1+x)^{-\frac{1}{4}}+\gamma x^{\frac{1}{5}}(1+x)^{-\frac{9}{20}} h(x, 0)} \\
& f^{\prime}(x, \infty) \rightarrow 0, h(x, \infty) \rightarrow 0
\end{aligned}
$$




\section{Numerical Method of Solution}

In the present investigation implicit finite difference method has been used to integrate Equations (12) to (13).

\subsection{Implicit Finite Difference Method}

We employed implicit finite difference method together with Keller box elimination technique, which was first introduced by Keller (1978) and widely used by Hossain et al. (1992, 1999).

To apply the aforementioned method, we first convert Equations (12) and (13) into the following system of first order equations with dependent variables $u(\xi, \eta), v(\xi, \eta), p(\xi, \eta)$ and $g(\xi, \eta)$ as

$$
\begin{aligned}
& f^{\prime}=u, u^{\prime}=v, g^{\prime}=p \\
& v^{\prime}+p_{1} f v-p_{2} u^{2}-p_{4} u+g=\xi\left(u \frac{\partial u}{\partial \xi}-v \frac{\partial f}{\partial \xi}\right) \\
& \frac{1}{\operatorname{Pr}} p^{\prime}+p_{1} f p-p_{3} u g+\frac{p_{5}}{\operatorname{Pr}} g p^{\prime}+\frac{p_{5}}{\operatorname{Pr}} p^{2}=\xi\left(u \frac{\partial g}{\partial \xi}-p \frac{\partial f}{\partial \xi}\right)
\end{aligned}
$$

where $\xi=\mathrm{x}, \mathrm{h}=\mathrm{g}$ and

$$
p_{1}=\frac{16+15 x}{20(1+x)}, p_{2}=\frac{6+5 x}{10(1+x)}, \quad p_{3}=\frac{1}{5(1+x)}, \quad p_{4}=M x^{\frac{2}{5}}(1+x)^{\frac{1}{10}}, p_{5}=\left(\frac{x}{1+x}\right)^{\frac{1}{5}} \gamma
$$

and the boundary conditions are

$$
\begin{aligned}
& f(\xi, 0)=0, u(\xi, 0)=0 \\
& p(\xi, 0)=\frac{\xi^{\frac{1}{5}}(1+\xi)^{-\frac{1}{5}} g(\xi, 0)-1}{(1+\xi)^{-\frac{1}{4}}+\gamma \xi^{\frac{1}{5}}(1+\xi)^{-\frac{9}{20}} g(\xi, 0)} \\
& u(\xi, 0)=0, g(\xi, 0)=0
\end{aligned}
$$

Now we consider the net rectangle on the $(\xi, \eta)$ plane shown in the Fig. 2 and denote the net points by

$$
\begin{aligned}
& \xi^{0}=0, \quad \xi^{n}=\xi^{n-1}+k_{n}, \quad n=1,2, \cdots \cdots \cdots \cdots, N \\
& \eta_{0}=0, \eta_{j}=\eta_{j-1}+h_{j}, \quad j=1,2, \cdots \cdots \cdots \cdots, J
\end{aligned}
$$

here $n$ and $j$ are just sequence of numbers on the $(\xi, \eta)$ plane, $k_{n}$ and $h_{j}$ are the variable mesh widths.

Approximate the quantities $f, u, v, p$ at the points $\left(\xi_{n}, \eta_{j}\right)$ of the net by $f_{j}^{n}, u_{j}^{n}, v_{j}^{n}, p_{j}^{n}$. which we call net function. We also employ the notation $p_{j n}$ for the quantities midway between net points shown in

Fig. 2 and for any net function as

$$
\begin{aligned}
& \xi^{n-1 / 2}=\frac{1}{2}\left(\xi^{n}+\xi^{n-1}\right) \\
& \eta_{j-1 / 2}=\frac{1}{2}\left(\eta_{j}+\eta_{j-1}\right)
\end{aligned}
$$


M. M. Rahman and M. A.Alim/ Journal of Naval Architecture and Marine Engineering 6(2009) 16-29

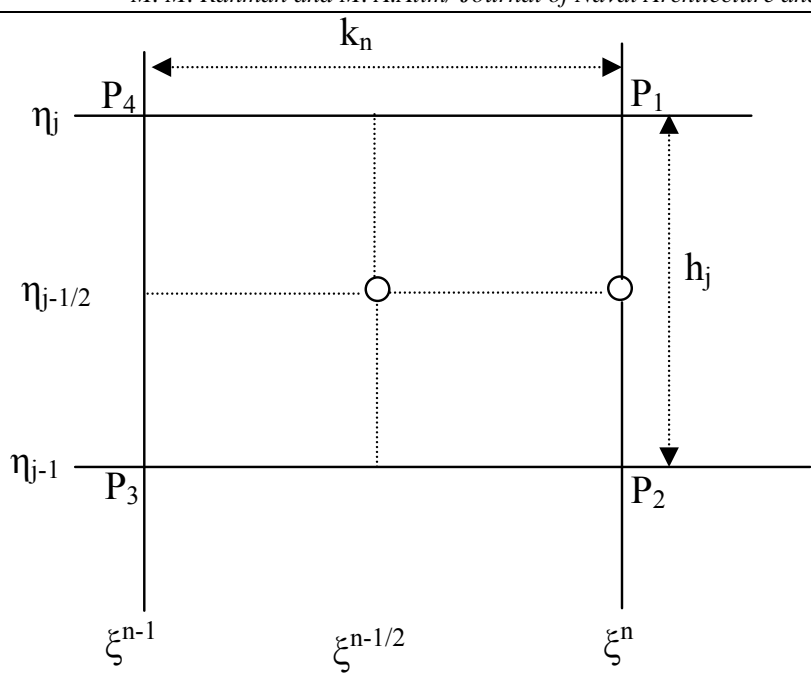

Fig. 2: Net rectangle for difference approximations for the Box scheme.

$g_{j}^{n-1 / 2}=\frac{1}{2}\left(g_{j}{ }^{n}+g_{j}^{n-1}\right)$

$g_{j-1 / 2}^{n}=\frac{1}{2}\left(g_{j}^{n}+g_{j-1}^{n}\right)$

The finite difference approximations according to box method to the three first order ordinary differential Equations (15) are written for the mid point $\left(\xi, \eta_{j-1 / 2}\right)$ of the segment $P_{1} P_{2}$ shown in the Fig. 2 and the finite difference approximations to the two first order differential equations (16) and (17) are written for the mid point $\left(\xi^{n-1 / 2}, \eta_{j-1 / 2}\right)$ of the rectangle $P_{1} P_{2} P_{3} P_{4}$. This procedure yields

$\frac{f_{j}^{n}-f_{j-1}^{n}}{h_{j}}=u_{j-1 / 2}^{n}=\frac{u_{j-1}^{n}+u_{j}^{n}}{2}$

$\frac{u_{j}^{n}-u_{j-1}^{n}}{h_{j}}=v_{j-1 / 2}^{n}=\frac{v_{j-1}^{n}+v_{j}^{n}}{2}$

$\frac{g_{j}^{n}-g_{j-1}^{n}}{h_{j}}=p_{j-1 / 2}^{n}=\frac{p_{j-1}^{n}+p_{j}^{n}}{2}$

The difference approximation to Equations (16)-(17) become

$$
\begin{aligned}
& h_{j}^{-1}\left(v_{j}^{n}-v_{j-1}^{n}\right)+\left\{p_{1}+\alpha_{n}\right\}(f v)_{j-1 / 2}^{n}-\left\{p_{2}+\alpha_{n}\right\}\left(u^{2}\right)_{j-1 / 2}^{n} \\
& -p_{4}(u)_{j-1 / 2}^{n}+g_{j-1 / 2}^{n}+\alpha_{n}\left(f_{j-1 / 2}^{n} v_{j-1 / 2}^{n-1}-v_{j-1 / 2}^{n} f_{j-1 / 2}^{n-1}\right)=R_{j-1 / 2}^{n-1}
\end{aligned}
$$

where $R_{j-1 / 2}^{n-1}=\alpha_{n}\left\{(f v)_{j-1 / 2}^{n-1}-\left(u^{2}\right)_{j-1 / 2}^{n-1}\right\}-L_{j-1 / 2}^{n-1}$

where $L_{j-1 / 2}^{n-1}=h_{j}^{-1}\left(v_{j}^{n-1}-v_{j-1}^{n-1}\right)-p_{1}(f v)_{j-1 / 2}^{n-1}-p_{2}\left(u^{2}\right)_{j-1 / 2}^{n-1}-p_{4}(u)_{j-1 / 2}^{n-1}+g_{j-1 / 2}^{n-1}$

where $\alpha_{n}=\frac{1}{k_{n}} \xi_{j-1 / 2}^{n-1 / 2}$ 


$$
\begin{aligned}
& \Rightarrow \frac{1}{P_{r}} h_{j}^{-1}\left(p_{j}^{n}-p_{j-1}^{n}\right)+\left(p_{1}+\alpha_{n}\right)(f p)_{j-1 / 2}^{n}-\left(p_{3}+\alpha_{n}\right)(u g)_{j-1 / 2}^{n} \\
& +\frac{p_{5}}{2 P_{r}} h_{j}^{-1} g_{j-1 / 2}^{n}\left(p_{j}^{n}-p_{j-1}^{n}+p_{j}^{n-1}-p_{j-1}^{n-1}\right)+\frac{p_{5}}{2 P_{r}} h_{j}^{-1} g_{j-1 / 2}^{n-1}\left(p_{j}^{n}-p_{j-1}^{n}\right) \\
& +\frac{p_{5}}{P_{r}}\left(p^{2}\right)_{j-\frac{1}{2}}^{n}+\alpha_{n}\left[u_{j-1 / 2}^{n} g_{j-1 / 2}^{n-1}-g_{j-1 / 2}^{n} u_{j-1 / 2}^{n-1}-p_{j-1 / 2}^{n} f_{j-1 / 2}^{n-1}+f_{j-1 / 2}^{n} p_{j-1 / 2}^{n-1}\right] \\
& =T_{j-1 / 2}^{n-1}
\end{aligned}
$$

where $T_{j-1 / 2}^{n-1}=-M_{j-1 / 2}^{n-1}+\alpha_{n}\left\{(f p)_{j-1 / 2}^{n-1}-(u g)_{j-1 / 2}^{n-1}\right\}$

The corresponding boundary conditions (18) become

$$
\begin{array}{ll}
f_{0}^{n}=0, & u_{0}^{n}=0, p_{0}^{n}(\xi, 0)=\xi^{\frac{1}{5}}(1+\xi)^{\frac{-1}{5}} g_{0}^{n}-1 /(1+\xi)^{\frac{-1}{4}}+\gamma \xi^{1 / 5}(1+\xi)^{\frac{-9}{20}} g_{0}^{n} \\
u_{j}^{n}=0, & g_{j}^{n}=0
\end{array}
$$

If assume $f_{j}^{n-1}, u_{j}^{n-1}, v_{j}^{n-1}, g_{j}^{n-1}, p_{j}^{n-1}$, to be known for $0 \leq j \leq J$, Equations (19) to (27) from a system of $5 \mathrm{~J}+5$ non-linear equations for the solutions of the $5 \mathrm{~J}+5$ unknowns $\left(f_{j}^{n}, u_{j}^{n}, v_{j}^{n}, g_{j}^{n}, p_{j}^{n}\right), \mathrm{j}=0,1,2,3, \ldots \ldots \mathrm{J}$.

These non-linear systems of algebraic equations are to be non-linearized by Newton's Quasi-linearization method. We define the iterates $\left(f_{j}^{n}, u_{j}^{n}, v_{j}^{n}, g_{j}^{n}, p_{j}^{n}\right), \mathrm{i}=0,1,2,3 \ldots$ IMAX with initial values equal those at the previous $x$-station. For the higher iterates thus the following form

$$
\begin{aligned}
& f_{j}^{(i+1)}=f_{j}^{i}+\delta f_{j}^{i} \\
& u_{j}^{(i+1)}=u_{j}^{i}+\delta u f_{j}^{i} \\
& v_{j}^{(i+1)}=v_{j}^{i}+\delta v_{j}^{i} \\
& g_{j}^{(i+1)}=g_{j}^{i}+\delta g_{j}^{i} \\
& p_{j}^{(i+1)}=p_{j}^{i}+\delta p_{j}^{i}
\end{aligned}
$$

Now by substituting the right hand sides of the above equations in place of $f_{j}^{n}, u_{j}^{n}, v_{j}^{n}$ and $g_{j}^{n}$ dropping the terms that are quadratic in $\delta f_{j}^{i}, \delta u_{j}^{i}, \delta v_{j}^{i}$ and $\delta P_{j}^{i}$ then the Equations (23), (24) and (26) in the following form

$$
\begin{aligned}
& f_{j}^{(i)}+\delta f_{j}^{(i)}-f_{j-1}^{(i)}-\delta f_{j-1}^{(i)}=\frac{h_{j}}{2}\left\{u_{j}^{(i)}+\delta u_{j}^{(i)}+u_{j-1}^{(i)}+\delta u_{j-1}^{(i)}\right\} \\
& \delta f_{j}^{(i)}-\delta f_{j-1}^{(i)}-\frac{h_{j}}{2}\left(\delta u_{j}^{(i)}+\delta u_{j-1}^{(i)}\right)=\left(r_{1}\right)_{j} \\
& \delta u_{j}^{(i)}-\delta u_{j-1}^{(i)}-\frac{h_{j}}{2}\left(\delta v_{j}^{(i)}+\delta v_{j-1}^{(i)}\right)=\left(r_{4}\right)_{j}
\end{aligned}
$$




$$
\begin{aligned}
& \delta g_{j}^{(i)}-\delta g_{j-1}^{(i)}-\frac{h_{j}}{2}\left(\delta p_{j}^{(i)}+\delta p_{j-1}^{(i)}\right)=\left(r_{5}\right)_{j} \\
& \left(s_{1}\right)_{j} \delta v_{j}^{(i)}+\left(s_{2}\right)_{j} \delta v_{j-1}^{(i)}+\left(s_{3}\right)_{j} \delta f_{j}^{(i)}+\left(s_{4}\right)_{j} \delta f_{j-1}^{(i)}+\left(s_{5}\right)_{j} \delta u_{j}^{(i)} \\
& +\left(s_{6}\right)_{j} \delta u_{j-1}^{(i)}+\left(s_{7}\right)_{j} \delta g_{j}^{(i)}+\left(s_{8}\right)_{j} \delta g_{j-1}^{(i)}+\left(s_{9}\right)_{j} .0+\left(s_{1}\right)_{j} .0=\left(r_{2}\right)_{j} \\
& \left(t_{1}\right)_{j} \delta p_{j}^{(i)}+\left(t_{2}\right)_{j} \delta p_{j-1}^{(i)}+\left(t_{3}\right)_{j} \delta f_{j}^{(i)}+\left(t_{4}\right)_{j} \delta f_{j-1}^{(i)}+\left(t_{5}\right)_{j} \delta u_{j}^{(i)} \\
& +\left(t_{6}\right)_{j} \delta u_{j-1}^{(i)}+\left(t_{7}\right)_{j} \delta g_{j}^{(i)}+\left(t_{8}\right)_{j} \delta g_{j-1}^{(i)}+\left(t_{9}\right)_{j} \delta v_{j}^{(i)}+\left(t_{10}\right)_{j} \delta v_{j-1}^{(i)}=\left(r_{3}\right)_{j} \\
& \left(r_{4}\right)_{j}=u_{j-1}^{(i)}-u_{j}^{(i)}+h_{j} v_{j-1 / 2}^{(i)} \\
& \left(r_{5}\right)_{j}=g_{j-1}^{(i)}-g_{j}^{(i)}+h_{j} p_{j-1 / 2}^{(i)} \\
& \left(r_{2}\right)_{j}=R_{j-1 / 2}^{n-1}-h_{j}^{-1}\left\{v_{j}^{(i)}-v_{j-1}^{(i)}\right\}-\frac{\left(P_{1}+\alpha_{n}\right)}{2}\left\{(f v)_{j}^{(i)}+(f v)_{j-1}^{i}\right\} \\
& +\frac{\left(P_{1}+\alpha_{n}\right)}{2}\left\{\left(u^{2}\right)_{j}^{(i)}+\left(u^{2}\right)_{j-1}^{(i)}\right\}+\frac{P_{4}}{2}\left\{u_{j}^{(i)}+u_{j-1}^{(i)}\right\}-\frac{1}{2}\left(g_{j}^{(i)}+g_{j-1 / 2}^{(i)}\right) \\
& -\frac{\alpha_{n}}{2}\left(f_{j}^{(i)}+f_{j-1}^{(i)}\right) v_{j-1 / 2}^{n-1}+\frac{\alpha_{n}}{2}\left(v_{j}^{(i)}+v_{j-1}^{(i)}\right) f_{j-1 / 2}^{n-1} \\
& \left(r_{3}\right)_{j}=T_{j-1 / 2}^{n-1}-\frac{1}{P_{r}} h_{j}^{-1}\left(p_{j}^{(i)}-p_{j-1}^{(i)}\right)-\frac{\left(P_{1}+\alpha_{n}\right)}{2}\left\{(f p)_{j}^{(i)}+(f p)_{j-1}^{(i)}\right\} \\
& +\frac{\left(P_{3}+\alpha_{n}\right)}{2}\left\{(u g)_{j}^{(i)}+(u g)_{j-1}^{(i)}\right\}-\frac{P_{5}}{2 \operatorname{Pr}} h_{j}^{-1}\left(g_{j}^{(i)}-g_{j-1}^{(i)}\right)\left\{P_{j}^{n}-P_{j-1}^{n}+P_{j}^{n-1}-P_{j-1}^{n-1}\right\} \\
& -\frac{P_{5}}{4 \operatorname{Pr}} h_{j}^{-1}\left(p_{j}^{(i)}-p_{j-1}^{(i)}\right) g_{j-1 / 2}^{n-1}-\frac{P_{5}}{4 \operatorname{Pr}}\left\{\left(p^{2}\right)_{j}^{(i)}+\left(p^{2}\right)_{j-1}^{(i)}\right\}-\frac{\alpha_{n}}{2}\left(u_{j}^{(i)}+u_{j-1}^{(i)}\right) g_{j-1 / 2}^{n-1} \\
& +\frac{\alpha_{n}}{2}\left(g_{j}^{(i)}-g_{j-1}^{(i)}\right) u_{j-1 / 2}^{n-1}+\frac{\alpha_{n}}{2}\left(p_{j}^{(i)}+p_{j-1}^{(i)}\right) f_{j-1 / 2}^{n-1}-\frac{\alpha_{n}}{2}\left(f_{j}^{(i)}+f_{j-1}^{(i)}\right) p_{j-1 / 2}^{n-1}
\end{aligned}
$$

The coefficients of momentum equation are

$$
\begin{aligned}
& \left(s_{1}\right)_{j}=h_{j}^{-1}+\frac{P_{1}+\alpha_{n}}{2} f_{j}^{(i)}-\frac{\alpha_{n}}{2} f_{j-1 / 2}^{n-1},\left(s_{2}\right)_{j}=-h_{j}^{-1}+\frac{P_{1}+\alpha_{n}}{2} f_{j}^{(i)}-\frac{\alpha_{n}}{2} f_{j-1 / 2}^{n-1} \\
& \left(s_{3}\right)_{j}=\frac{\left(P_{1}+\alpha_{n}\right)}{2} v_{j}^{(i)}+\frac{\alpha_{n}}{2} v_{j-1 / 2}^{n-1},\left(s_{4}\right)_{j}=\frac{\left(P_{1}+\alpha_{n}\right)}{2} v_{j-1}^{(i)}+\frac{\alpha_{n}}{2} v_{j-1 / 2}^{n-1} \\
& \left(s_{5}\right)_{j}=-\left(P_{1}+\alpha_{n}\right) u_{j}^{(i)}-\frac{P_{4}}{2},\left(s_{6}\right)_{j}=-\left(P_{1}+\alpha_{n}\right) u_{j-1}^{(i)}-\frac{P_{4}}{2} \\
& \left(s_{7}\right)_{j}=[1 / 2],\left(s_{8}\right)_{j}=[1 / 2],\left(s_{9}\right)_{j}=0,\left(s_{10}\right)_{j}=0
\end{aligned}
$$

The coefficients of energy equation are

$$
\left(t_{1}\right)_{j}=\frac{1}{\operatorname{Pr}} h_{j}^{-1}+\frac{\left(p_{1}+\alpha_{n}\right)}{2} f_{j}^{(i)}+\frac{p_{5}}{2 \operatorname{Pr}} h_{j}^{-1} g_{j-1 / 2}^{n-1}+\frac{p_{5}}{\operatorname{Pr}}-\frac{\alpha_{n}}{2} f_{j-1 / 2}^{n-1}
$$




$$
\begin{aligned}
& \left(t_{2}\right)_{j}=-\frac{1}{\operatorname{Pr}} h_{j}^{-1}+\frac{\left(p_{1}+\alpha_{n}\right)}{2} f_{j-1}^{(i)}-\frac{p_{5}}{2 \operatorname{Pr}} h_{j}^{-1} g_{j-1 / 2}^{n-1}+\frac{p_{5}}{\operatorname{Pr}}-\frac{\alpha_{n}}{2} f_{j-1 / 2}^{n-1} \\
& \left(t_{3}\right)_{j}=\frac{\left(p_{1}+\alpha_{n}\right)}{2} p_{j}^{(i)}+\frac{\alpha_{n}}{2} p_{j-1 / 2}^{n-1},\left(t_{4}\right)_{j}=\frac{\left(p_{1}+\alpha_{n}\right)}{2} p_{j-1}^{(i)}+\frac{\alpha_{n}}{2} p_{j-1 / 2}^{n-1} \\
& \left(t_{5}\right)_{j}=-\frac{\left(p_{3}+\alpha_{n}\right)}{2} g_{j}^{(i)}+\frac{\alpha_{n}}{2} g_{j-1 / 2}^{n-1},\left(t_{6}\right)_{j}=-\frac{\left(p_{3}+\alpha_{n}\right)}{2} g_{j-1}^{(i)}+\frac{\alpha_{n}}{2} g_{j-1 / 2}^{n-1} \\
& \left(t_{7}\right)_{j}=-\frac{\left(p_{3}+\alpha_{n}\right)}{2} u_{j}^{(i)}+\frac{p_{5}}{4 \operatorname{Pr}} h_{j}^{-1}\left\{P_{j}^{n}-P_{j-1}^{n}+P_{j}^{n-1}-P_{j-1}^{n-1}\right\}-\frac{\alpha_{n}}{2} u_{j-1 / 2}^{n-1} \\
& \left(t_{8}\right)_{j}=-\frac{\left(p_{3}+\alpha_{n}\right)}{2} u_{j-1}^{(i)}+\frac{p_{5}}{4 \operatorname{Pr}} h_{j}^{-1}\left\{P_{j}^{n}-P_{j-1}^{n}+P_{j}^{n-1}-P_{j-1}^{n-1}\right\}-\frac{\alpha_{n}}{2} u_{j-1 / 2}^{n-1} \\
& \left(t_{9}\right)_{j}=0,\left(t_{10}\right)_{j}=0
\end{aligned}
$$

The boundary conditions (28) become

$$
\begin{aligned}
& \delta f_{0}^{n}=0, \quad \delta u_{0}^{n}=0, \quad \delta p_{0}^{n}(\xi, 0)=\delta\left[\xi^{\frac{1}{5}}(1+\xi)^{\frac{-1}{5}} g_{0}^{n}-1 /(1+\xi)^{\frac{-1}{4}}+\gamma \xi^{1 / 5}(1+\xi)^{\frac{-9}{20}} g_{0}^{n}\right] \\
& \delta u_{j}^{n}=0, \quad \delta g_{j}^{n}=0
\end{aligned}
$$

Which just express the requirement for the boundary conditions to remain during the iteration process. Now the system of linear equations (34), (35), (36), (37) and (38) together with the boundary conditions (41) can be written in a black matrix form a coefficient matrix. The whole procedure, namely reduction to first order followed by central difference approximations, Newton's Quasi-linearization method and the block Thomas algorithm, is well known as Keller-box method.

\section{Local skin friction coefficient and surface temperature distribution}

From the process of numerical computation, in practical point of view, it is important to calculate the values of the surface shear stress in terms of the skin friction coefficient. This can be written in the non-dimensional form as

$$
C_{f}=\left(G r^{-3 / 4} l^{2}\right) /(\mu v) \tau_{w}
$$

where $\tau_{w}\left[=\mu(\partial \bar{u} / \partial \bar{y})_{\bar{y}=0}\right]$ is the shearing stress. Using the new variables described in Equation (6), the local skin friction co-efficient can be written as

$$
C_{f x}=x^{2 / 5}(1+x)^{-3 / 20} f^{\prime \prime}(x, 0)
$$

The numerical values of the surface temperature distribution are obtained from the relation

$$
\theta(x, 0)=x^{1 / 5}(1+x)^{-1 / 5} h(x, 0)
$$

We have also discussed the velocity profiles and the temperature distributions for different values of the thermal conductivity variation parameter, the magnetic parameter and the Prandtl number.

\section{Comparison with previous work and programme validation}

A comparison of the surface temperature and local skin friction coefficient obtained in the present work with $x^{\frac{1}{5}}=\xi, M=0$ and $\gamma=0$ and obtained by Merkin and Pop (1996) and Pozzi and Lupo (1988) have been shown in Table 1 and Table 2, respectively. It is clearly seen that there is an excellent agreement among the respective results. 
M. M. Rahman and M. A.Alim/ Journal of Naval Architecture and Marine Engineering 6(2009) 16-29

Table 1: Comparison of the present numerical results of surface temperature with Prandtl number $\quad P r=$ $0.733, M=0$ and $\gamma=0$

\begin{tabular}{|l|l|l|l|}
\hline \multicolumn{2}{|c|}{$\theta(x, 0)$} & Present work \\
\hline$x^{\frac{1}{5}}=\xi$ & Pozzi and Lupo (1988) & Merkin and Pop (1996) & \\
\hline 0.7 & 0.651 & 0.651 & 0.651 \\
\hline 0.8 & 0.684 & 0.686 & 0.687 \\
\hline 0.9 & 0.708 & 0.715 & 0.716 \\
\hline 1.0 & 0.717 & 0.741 & 0.741 \\
\hline 1.1 & 0.699 & 0.762 & 0.763 \\
\hline 1.2 & 0.640 & 0.781 & 0.781 \\
\hline
\end{tabular}

Table 2: Comparison of the present numerical results of local skin friction coefficient with Prandtl number $\mathrm{Pr}=$ $0.733, M=0$ and $\gamma=0$

\begin{tabular}{|l|l|l|l|}
\hline \multicolumn{2}{|l|}{$C_{f_{x}}$} \\
\hline$x^{\frac{1}{5}}=\xi$ & Pozzi and Lupo (1988) & Merkin and Pop (1996) & Present work \\
\hline 0.7 & 0.430 & & \\
\hline 0.8 & 0.530 & 0.430 & 0.424 \\
\hline 0.9 & 0.635 & 0.530 & 0.529 \\
\hline 1.0 & 0.741 & 0.635 & 0.635 \\
\hline 1.1 & 0.829 & 0.745 & 0.744 \\
\hline 1.2 & 0.817 & 0.859 & 0.860 \\
\hline
\end{tabular}

\section{Results and Discussion}

Here we have investigated numerically MHD free convection flow along a vertical flat plate with temperature dependent thermal conductivity and heat conduction. In simulation, the values of the Prandtl number are considered to be $0.70,1.70,2.97$ and 4.34 that corresponds to helium, sulfur dioxide, methyl chloride and water, respectively. Numerical results have been obtained for different values of the magnetic parameter, thermal conductivity variation parameter and Prandtl number are presented graphically.

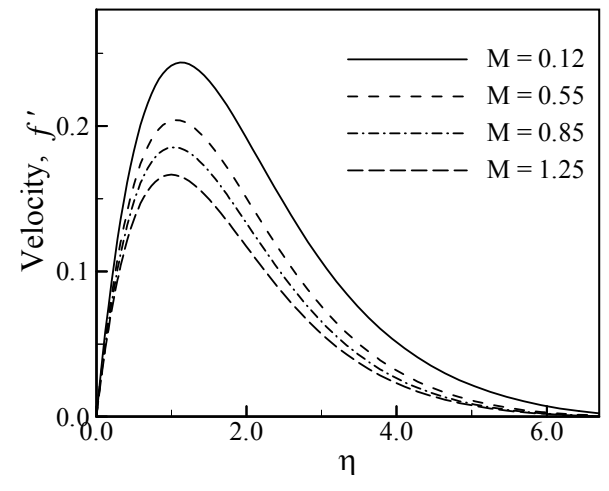

(a)

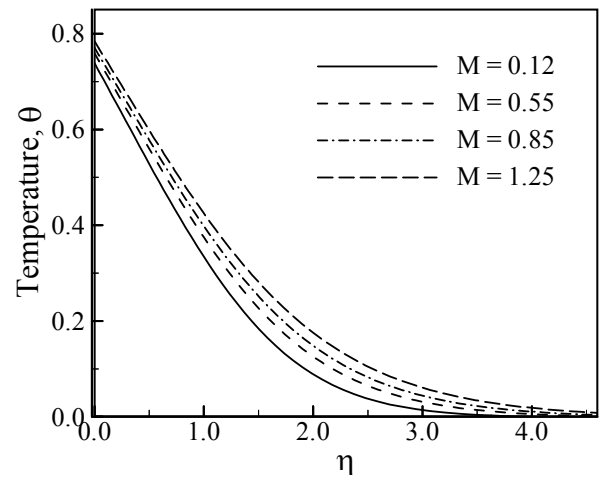

(b)

Fig: 3 (a) Variation of velocity profiles and (b) temperature profiles against $\eta$ for varying of $M$ with $\gamma=0.12$ and $\operatorname{Pr}=4.34$ 


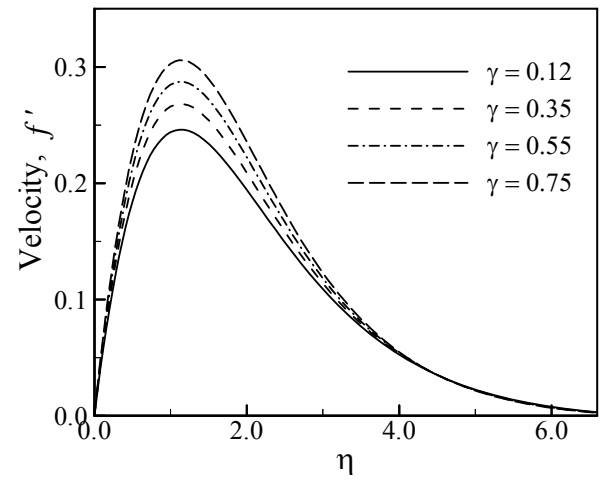

(a)

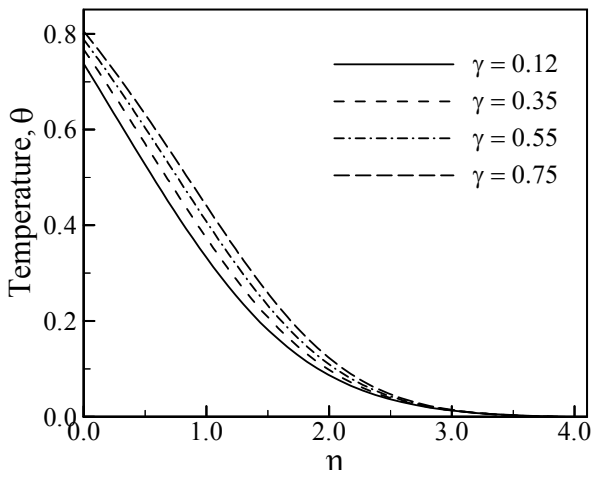

(b)

Fig. 4 (a) Variation of velocity profiles and (b) temperature profiles against $\eta$ for varying of $\gamma$ with $M=0.12$ and $P r=4.34$

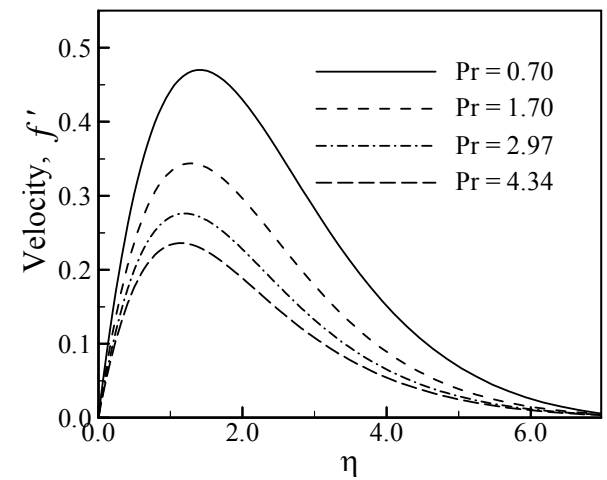

(a)

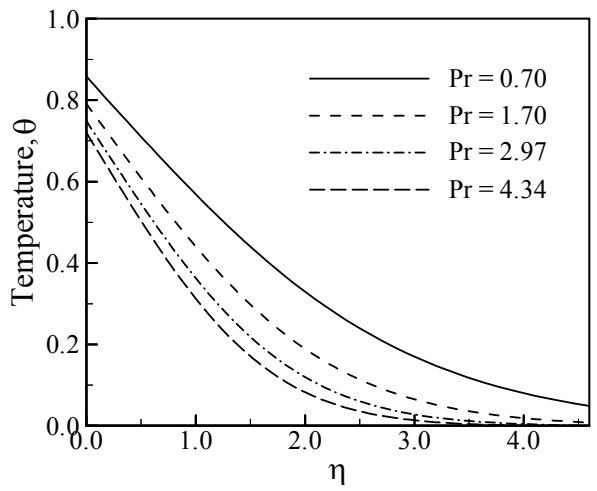

(b)

Fig. 5 (a) Variation of velocity profiles and (b) temperature profiles against $\eta$ for varying of $\operatorname{Pr}$ with $\gamma=0.12$ and $M=0.12$

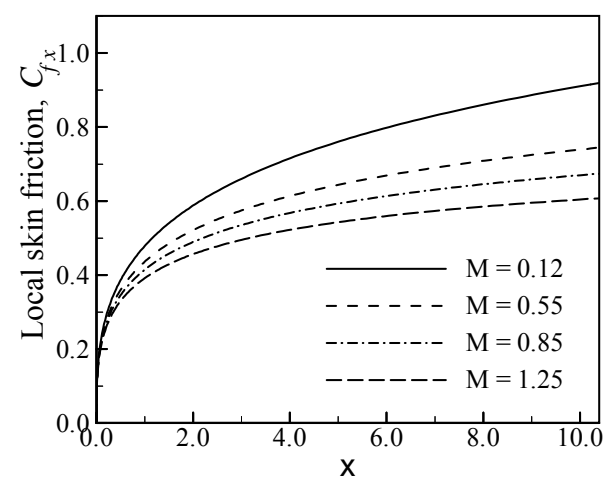

(a)

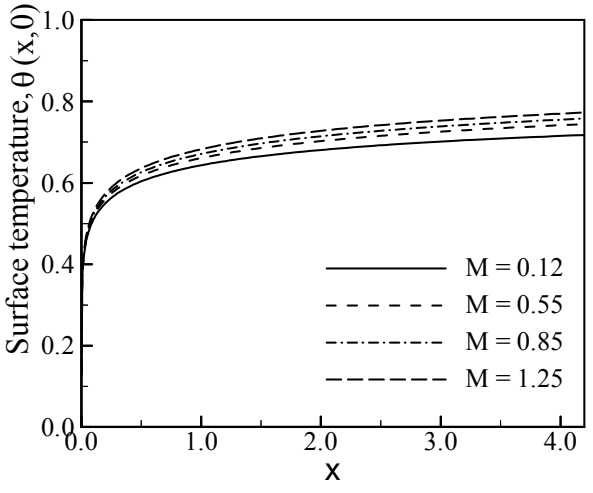

(b)

Fig. 6 (a) Variation of local skin friction coefficients and (b) surface temperature distribution profiles against $x$ for varying of $M$ with $\gamma=0.12$ and $\operatorname{Pr}=4.34$ 


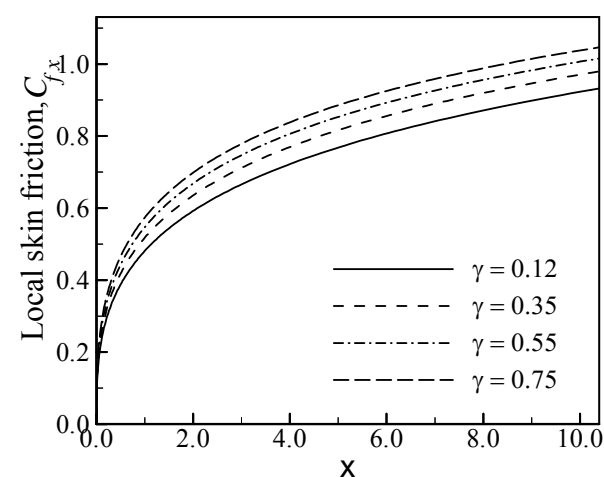

(a)

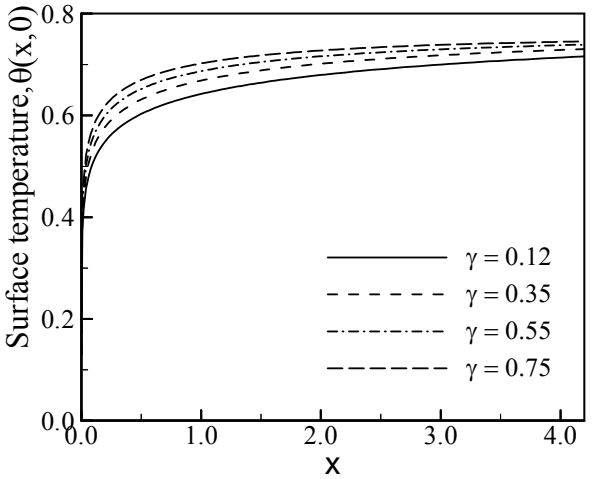

(b)

Fig. 7 (a) Variation of local skin friction coefficients and (b) surface temperature distribution profiles against $x$ for varying of $\gamma$ with $M=0.12$ and $\operatorname{Pr}=4.34$

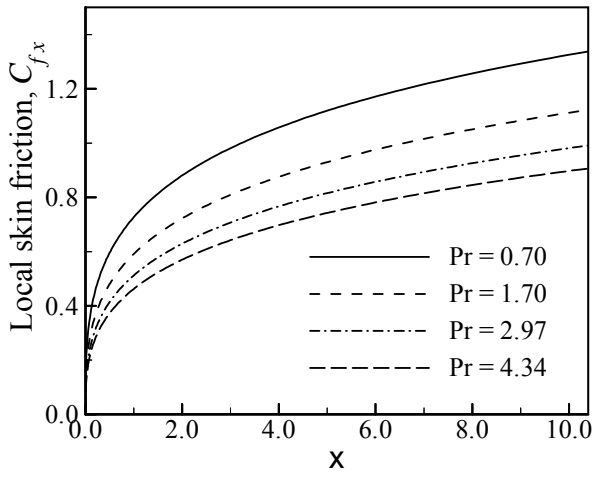

(a)

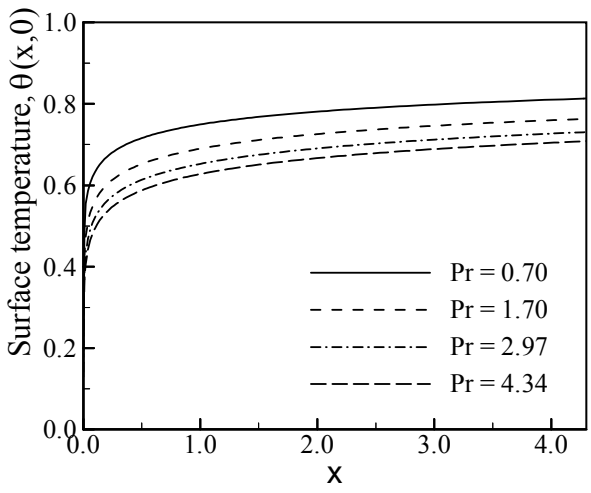

(b)

Fig. 8 (a) Variation of local skin friction coefficients and (b) surface temperature distribution profiles against $x$ for varying of $\operatorname{Pr}$ with $\gamma=0.12$ and $M=0.12$

From Fig. 3 (a) it can be observed that the magnetic field normal to the flow in an electrically conducting fluid introduces a Lorentz force, which acts against the flow. The peak velocity decreases with the increase in magnetic parameter $M$ due to this retarding effect. From Fig. 3 (b), it can be seen that the temperature within the boundary layer increases with the increase in $M$. Temperature at the interface also varies since the conduction is considered with in the plate.

Fig. 4 (a) and 4(b) display the numerical results of the velocity and the temperature, respectively obtained from the solution of the Equations (12) and (13) subject to the boundary condition (14) for different values of thermal conductivity variation parameter $\gamma$ plotted against $\eta$ with $M=0.12$ and $\operatorname{Pr}=4.34$. It is seen from Fig. 4(a) and Fig.4 (b) that the velocity and temperature increase within the boundary layer with the increasing value of $\gamma$. It means that the velocity boundary layer and the thermal boundary layer thickness increase for increasing values of $\gamma$.

Fig. 5 (a) and Fig. 5 (b) illustrate the velocity and temperature profiles for different values of Prandtl number $P r$ with $M=0.12$ and $\gamma=0.12$. From Fig. 5 (a), it can be observed that the velocity decreases as well as its position moves toward the interface with the increase in Pr. From Fig. 5 (b), it is seen that the temperature profiles shift downward with the increasing values of $\mathrm{Pr}$.

The variation of the local skin friction coefficient $C_{f x}$ and surface temperature $\theta(x, 0)$ for different values of $M$ with $\gamma=0.12$, and $\operatorname{Pr}=4.34$ at different positions are illustrated in Fig. 6. (a) and Fig.6 (b), respectively. It is observed from Fig. 6. (a) that the increase in value of the magnetic parameter $M$ leads to a decrease in the skin friction factor. Again Fig. 6 (b) shows that the surface temperature $\theta(x, 0)$ increases due to the increased value of the magnetic parameter $M$. 
Fig. 7 (a) and Fig. 7 (b) illustrate the effect of the thermal conductivity variation parameter on the local skin friction coefficient and surface temperature distribution against $x$ with $M=0.12$ and $\operatorname{Pr}=4.34$. It is also seen that the local skin friction coefficient increases with the increase in $\gamma$. From Fig. 7 (b), it is seen that the surface temperature increases with the increase in $\gamma$. This is to be expected because the higher value for the thermal conductivity variation parameter accelerates the fluid flow and increases the temperature as mentioned in Fig. 4 (a) and Fig. 4 (b), respectively.

Fig. 8 (a) and Fig. 8 (b) plot the effect of Prandtl number on the local skin friction coefficient and surface temperature distribution against $x$ with $M=0.12$ and $\gamma=0.12$. It can be observed from Fig. 8 (a) that the local skin friction coefficient decreases with the increase in $\mathrm{Pr}$. This is expected behavior because the fluid velocity is decreased due to the increase in $\operatorname{Pr}$. From Fig. 8 (b), it can be conclude that the surface temperature distribution decreases for the increase in values of $\mathrm{Pr}$.

\section{Conclusion}

In this paper effect of temperature dependent thermal conductivity on MHD free convection flow along a vertical flat plate have been studied numerically. Implicit finite difference method together with Keller box scheme is employed to integrate the equations governing the flow. Comparison with previously published work is performed and excellent argument has been observed. From the present numerical investigation, following conclusions may be drawn:

- For increased value of magnetic parameter, the velocity profile decreases but the temperature profile increases slightly.

- The local skin friction coefficient decreases as well as the surface temperature distribution increase with the increase in values of the magnetic parameter.

- The velocity and the temperature within the boundary layer increases for increasing values of the thermal conductivity variation parameter.

- Increasing values of the thermal conductivity variation parameter leads to increase the local skin friction coefficient and also the surface temperature distribution.

- The velocity, temperature, local skin friction coefficient and surface temperature distribution within the boundary layer decreases with the increase in values of the Prandtl number.

\section{Reference}

Arunachalam, M. and Rajappa, N.R., (1978): Thermal boundary layer in liquid metals with variable thermal conductivity, Appl. Sci. Res., Vol. 34, pp.179-187. doi:10.1007/BF00418866

Charraudeau, J., (1975): Influence de gradients de properties physiques en convection force application au cas du tube, Int. J. Heat Mass Trans., Vol.18, pp.87-95. doi:10.1016/0017-9310(75)90011-3

Cheng, L. C. A., (2006): Numerical simulation of micro polar fluid flows along a flat plate with wall conduction and buoyancy effects, J. Appl. Phys. D, Vol.39, pp.1132-1140. doi:10.1088/0022-3727/39/6/019

Chowdhury, M. K. and Islam, M. N., (2000): MHD free convection flow of visco-elastic fluid past an infinite porous plate, Int. J. Heat Mass Trans., Vol.36, pp. 439-447. doi:10.1007/s002310000103

Elbashbeshy, E. M. A., (2000): Free convection flow with variable viscosity and thermal diffusivity along a vertical plate in the presence of magnetic field, Int. J. Eng. Sci., Vol.38, pp.207-213. doi:10.1016/S00207225(99)00021-X

Hossain, M. A. and Ahmad, M., (1990): MHD forced and free convection boundary layer flow near the leading edge, Int. J. Heat Mass Trans., Vol.33, No.3, pp.571-575. doi:10.1016/0017-9310(90)90190-6

Hossain, M. A., (1992): The viscous and Joule heating effects on MHD free convection flow with variable plate temperature, Int. J. Heat Mass Trans., Vol.35, No.12, pp. 3485-3487. doi:10.1016/0017-9310(92)90234-J 
Hossain, M.A., Alam, K.C.A. and Rees, D.A.S., (1997): MHD forced and free convection boundary layer flow along a vertical porous plate, Applied Mechanics and Engineering, Vol.2, No.1, pp.33-51. Hossain, M.A., Das, S. K. and Pop, I., (1998): Heat transfer response of MHD free convection flow along a vertical plate to surface temperature oscillation, Int. J. Non-Linear Mechanics, Vol. 33, No.3, pp.541-553. doi:10.1016/S0020-7462(96)00151-5

Hossain, M. A., Alim, M. A. and Rees, D. A. S., (1999): The effect of radiation on free convection from a porous vertical plate, Int. J. Heat Mass Trans., Vol.42, pp.181-191. doi:10.1016/S0017-9310(98)00097-0

Hossain, M.A., Munir, M.S.and Gorla, R.S.R., (2002): Combined convection from a vertical flat plate with temperature dependent viscosity and thermal conductivity, Int. J. Fluid Mech.Res., Vol.29, No.6, pp.725-741.

Hossain, M.A., Molla, M.M. and Rees, D.A.S., (2004): Natural convection laminar flow with temperature dependent viscosity and thermal conductivity along a vertical wavy surface, Int. J. Heat and Fluid flow, (Communicating)

Keller, H.B., (1978): Numerical methods in boundary layer theory, Annual Rev. Fluid Mechanics, Vol.10, pp.417-433. doi:10.1146/annurev.fl.10.010178.002221

Kuiken, H. K., (1970): Magnetohydrodynamic free convection in strong cross flow field, J. Fluid Mech., Vol.40, pp.21-38. doi:10.1017/S0022112070000022

Luikov, A. K., (1974): Conjugate convective heat transfer problems. Int. J. Heat Mass Trans., Vol.16, pp. 57265.

Molla, M.M., Rahman, A. and Lineeya, L.T., (2005): Natural convection flow from an isothermal sphere with temperature dependent thermal conductivity, J. Arc. Marine Eng., Vol.2, pp.53-64.

Merkin, J. H. and Pop, I., (1996): Conjugate free convection on a vertical surface, Int. J. Heat Mass Trans., Vol.39, pp.1527-1534. doi:10.1016/0017-9310(95)00238-3

Pop, I. and Ingham, D. B., (2001): Convective heat transfer, (Pergamon, Oxford). p. 179. doi:10.1016/B978$\underline{008043878-8 / 50009-2}$

Pozzi, A. and Lupo, M., (1988): The coupling of conduction with laminar natural convection along a flat plate, Int. J. Heat Mass Trans., Vol.31, No. 9, pp.1807-1814. doi:10.1016/0017-9310(88)90195-0

Sparrow, E. M. and Cess, R. D., (1961): Effect of magnetic field on free convection heat transfer, Int. J. Heat Mass Trans., Vol.3, p.267. doi:10.1016/0017-9310(61)90042-4 